\title{
Cooperative Transmission Strategy Over Users' Mobility for Downlink Distributed Antenna Systems
}

\author{
Ashim Khadka*, Koichi Adachi ${ }^{\dagger}$, Sumei Sun ${ }^{\ddagger}$, Junyuan Wang *, Huiling Zhu* and Jiangzhou Wang* \\ *School of Engineering and Digital Arts, University of Kent, United Kingdom \\ ${ }^{\dagger}$ Advanced Wireless and Communication Research Center, The University of Electro-Communications, Japan \\ ${ }^{\ddagger}$ Institute for Infocomm Research, A*STAR, Singapore
}

\begin{abstract}
Previously, a scheme in [1] is proposed for the outdated channel state information (CSI) problem, for data transmission in time division duplex (TDD) systems. In user movement environment, the actual channel of data transmission at downlink time slot is different from the estimated channel due to channel variation. In this paper the effect of different user mobility on TDD downlink multiuser distributed antenna system is investigated. An efficient autocorrelation based feedback interval technique is proposed and updates CSI at less cost of the downlink time slots. In the proposed technique, the frequency of CSI feedback for different users is proportional to their speed. Cooperative clusters are formed to maximize sum rate where channel gain based antenna selection and user clustering based on SINR threshold is applied to reduce computational complexity. Numerical results show that sum rate superiority of the proposed scheme over the user mobility.
\end{abstract}

\section{INTRODUCTION}

A $\mathrm{S}$ the demand of the multimedia applications grows in wireless communication systems, high data rate and extended coverage will form the foundation for future services[1],[3]. Distributed antenna system (DAS) is a very promising technique to efficiently extend coverage area and reduce overall transmit power by reducing the distance between the transmitter and the receiver [4]-[8]. In the DAS, a number of remote antenna units (RAUs) are deployed at geographically separated locations and controlled by a single central unit (CU) via optical fibre or cable [9], [10]. All RAUs have different independent channel characteristics because the signal from RAU to different users experience different large scale fading and different small scale fading. Multi-user transmission supported by the DAS causes inter-user interference (IUI), which is considered as a key limiting factor in wireless communication systems. A linear precoding like zero forcing (ZF) and minimum mean square error (MMSE) [11]-[13] are used to mitigate the IUI. The precoding technique as zero forcing uses channel state information (CSI) from all users at the $\mathrm{CU}$ to form noninterfering beams.

The increasing demand of high data rate due to the use of multimedia services, require asymmetric traffic between uplink and downlink. The asymmetric traffic in frequency division duplex (FDD) system requires extra system bandwidth, whereas time division duplex (TDD) can fit it into any single spectrum by allocating uplink and downlink time slots according to the traffic condition [14], [15]. In TDD system, the CU estimates CSI from the uplink pilot sent by the users at uplink time slot and then uses it via channel reciprocals to generate transmit

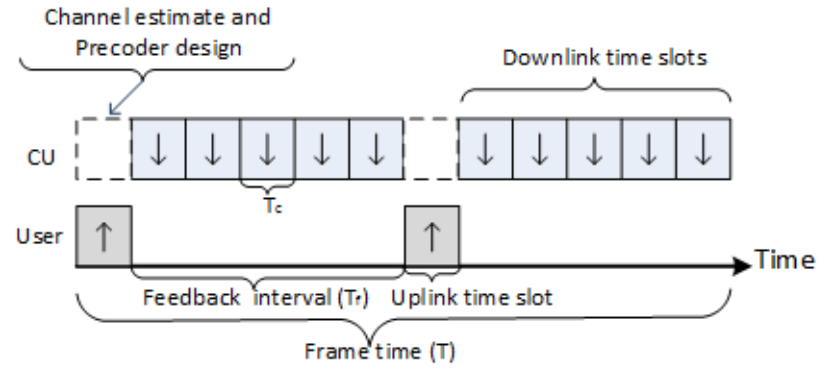

Fig. 1. Illustration of feedback interval.

precoding matrix for downlink transmission at the downlink time slot [16]. However, the CSI obtained by the CU is outdated in practice due to channel variation in user movement environment.

When the user moves, the channel from RAU to the user becomes a time varying channel. In this situation, the transmitted signal is subject to the Doppler effect and hence experiences frequency offset. When the mobility speed increases or the transmission delay increases, the correlation between the actual channel and the estimated channel becomes small which severely degrades the performance. For a multi-user system with linear precoding, the outdated CSI mismatch the actual channel and the precoder. In [17], as the interference increases and the performance of the precoding system degrades due to the degradation of the channel correlation. In [18], the performance of the multiuser MIMO system strongly depends on the correlation between the real channel and the estimated CSI at the transmitter. In [19], the multiuser MIMO system is considered in the presence of non-perfect CSI where only low mobility users are jointly served and space time coding transmission is allocated to high mobility users.

The low mobility user implicitly has high channel temporal correlation coefficient within a feedback interval because of slow channel variation. The feedback interval is the time duration of two uplink time slots where the CU estimates the CSI as shown in fig. 1. For high mobility user, the channel mismatch becomes large because the channel varies fast. This channel mismatch can be reduced by reducing feedback interval. By reducing the feedback interval in the TDD mode, the uplink transmission increases at the expenses of reduced downlink transmission in the overall time slots. In [20] and [21], users are divided into several groups based on their channel strength and number of feedback timeslot is allocated to each group.

In this paper, we investigate the effect of user mobility 
on the system performance and propose an efficient feedback reduction technique for downlink multiuser DAS. The proposed technique divides users into several groups, where feedback interval is allocated based on mobility state information (MSI) as speed. In the DAS, due to less propagation loss between RAU and user, we consider antenna selection of RAUs and interference based user clustering. Even though same the structure of the feedback reduction technique of [1] is utilized, our work is distinguished from previous work [1] for the following reasons: 1) The channel mismatch error is introduced in our system model, which is controlled by setting threshold values. Therefore, the channel estimation can be fixed by simply changing the threshold values. 2) The superiority of our proposed scheme increases as the user speed increases. This is due to individual threshold value allocation to each cluster and the length of feedback interval increases in compare to previous technique in [1].

\section{SySTEM MODEL}

Consider a single rectangular cell downlink environment which consists of $N_{t}$ RAUs and $K$ users, as shown in Fig. 2. Each user is equipped with a single antenna. The users are uniformly distributed within a cell. We assume that $N_{t} \geq K$, the CU perfectly estimates the CSI of all users at the uplink transmit slot and the MSI of the user is also perfectly known at the CU. Let $\mathcal{K}$ denote the user set, i.e., $\mathcal{K}=\{1, \cdots, \cdots, K\}$ and $\mathcal{N}$ denote the RAU set, i.e., $\mathcal{N}=\left\{1, \cdots, \cdots, N_{t}\right\}$. Under these assumptions, the received signal for the $k$-th user at time $t$ is given by:

$$
\begin{aligned}
y_{k}(t) & =\mathbf{h}^{k}(t) \mathbf{w}_{k}(t-\tau) \sqrt{p_{k}(t-\tau)} s_{k}(t-\tau) \\
& +\mathbf{h}^{k}(t) \sum_{i \in \mathcal{K}, i \neq k} \mathbf{w}_{i}(t-\tau) \sqrt{p_{i}(t-\tau)} s_{i}(t-\tau)+n_{k}(t)
\end{aligned}
$$

where $\mathbf{h}^{k} \in \mathbb{C}^{1 \times N_{t}}$ is a time-varying channel vector, $\mathbf{w}_{k} \in$ $\mathbb{C}^{N_{t} \times K}$ denotes the precoding vector, $p_{k}$ is a power normalization factor of each $\mathrm{RAU}, \mathbf{s} \in \mathbb{C}^{K \times 1}$ is a transmit symbol vector and $n_{k} \sim \mathcal{C N}\left(0, \sigma_{k}^{2}\right)$ is the additive White Gaussian noise (AWGN). The $(1, j)$-th element of the channel vector $\mathbf{h}^{\mathbf{k}}(t)$ represent the channel from RAU $j$ to user $k$ at time $t$, i.e, $h_{k, j}(t)$, which consists of path loss and Rayleigh fading and is given as

$$
h_{k, j}(t)=l_{k, j}(t) \cdot \tilde{h}_{k, j}(t)
$$

where $l_{k, j}(t)=d_{k, j}^{-\frac{\alpha}{2}}(t)$ denotes the propagation path loss with path loss exponent $\alpha$ and distance $d_{k, j}$ between user $k$ and RAU $j$ at time $t, \tilde{h}_{k, j}(t)$ is small scale fading from RAU $j$ to user $k$ at time $t$ and is independently and identically distributed. The $\tilde{h}_{k, j}(t)$ is Rayleigh distributed and modelled as Jakes fading model [22] where $N_{0}$ scatters arrive at moving user with uniformly distributed arrival angles $\alpha_{n}$, such that scatter $n$ experiences a Doppler shift $\omega_{n}=\frac{2 \pi f_{c} v}{c} \cos \alpha_{n}$ where $f_{c}$ is the carrier frequency, $v$ is the user speed, $c$ is the speed of light. The small scale fading is given as

$\tilde{h}_{k, j}(t)=\sqrt{\frac{2}{N_{0}}} \sum_{n=1}^{N_{0}} \mathbf{A}_{j}(n)\left[\cos \left(\beta_{n}\right)+i \sin \left(\beta_{n}\right)\right] \cos \left(\omega_{n} t+\theta_{n}\right)$

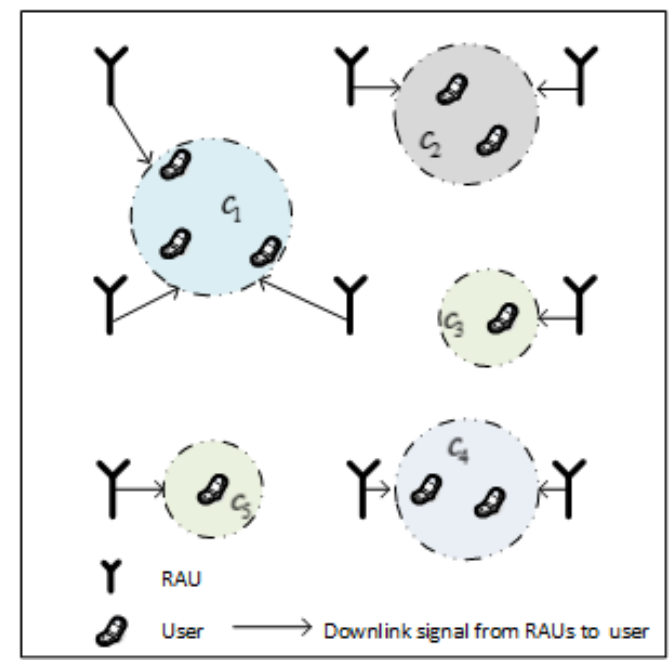

Fig. 2. DAS architecture in cell.

where $\mathbf{A}_{j}(n)$ is an orthogonal vector of Walsh-Hadamard codewords to generate multiple uncorrelated waveforms at moving user, $\beta_{n}=\frac{\pi n}{N_{0}}$ is a phase and gives zero correlation between the real and imaginary parts of $h_{k, i}(t), \theta_{n}$ is oscillator phase. The arrival angles $\alpha_{n}=\pi(n-0.5) / 2 N_{0}$.

In the TDD system, there exists a delay $\tau$ from the instant when CSI is obtained to downlink transmission. Taking into account the fact that large scale fading change much slower than the small scale fading, we modify the model in [23] and is given as:

$$
\mathbf{h}^{k}(t)=\rho \mathbf{h}^{k}(t-\tau)+\sqrt{\left(1-\rho^{2}\right)} \mathbf{e}^{k}(t)
$$

where $\mathbf{h}^{k}(t-\tau)=\mathbf{l}_{k}(t-\tau) \cdot \tilde{\mathbf{h}}^{k}(t-\tau)$ is the estimated channel vector, where its element is obtained from (3), e $(t)=$ $\mathbf{l}_{k}(t-\tau) \cdot \tilde{\mathbf{e}}^{k}(t)$ is the error in the estimate that is uncorrelated with $\hat{\mathbf{h}}^{k}$ and $\rho$ is the correlation coefficient between the actual channel gain and its estimate [24], which is given by $\rho=\mathbb{E}\left[h_{k, j}(t) \hat{h}_{k, j}(t-\tau)\right] / \sqrt{\mathbb{E}\left[\left|h_{k, j}(t)\right|^{2}\left|\hat{h}_{k, j}(t-\tau)\right|^{2}\right]}=$ $J_{0}\left(2 \pi f_{d} \tau\right)$, where $J_{0}(\cdot)$ is the zeroth-order Bessel function of the first kind and $f_{d}=\frac{v}{c} f_{c}$.

For a TDD system, the CU designed the precoding matrix based on the estimated channel at time $t-\tau$. Zero-forcing precoding is considered which completely eliminates interference, i.e., $\mathbf{h}^{k}(t-\tau) \mathbf{w}_{i}(t-\tau)=0 \forall i \in \mathcal{K} \backslash\{k\}$. The precoding matrix $\mathbf{W}$ is the pseudoinverse of $\hat{\mathbf{H}}$ [12], i.e.,

$$
\mathbf{W}(t-\tau)=\mathbf{H}^{H}(t-\tau)\left(\mathbf{H}(t-\tau) \mathbf{H}^{H}(t-\tau)\right)^{-1}
$$

For a non-zero delay $(\tau \neq 0)$, the CSI is imperfect and $\mathbf{h}^{k}(t) \mathbf{w}_{i}(t-\tau)=\sqrt{\left(1-\rho^{2}\right)} \mathbf{e}^{k}(t) \mathbf{w}_{i}(t-\tau)$. Due to this mismatch, the desired user symbol is interfered with the other users' symbols due to the presence of residual interference.

The received SINR of user $k$ at time $t$ is given by

$$
\gamma_{k}(t)=\frac{p_{k}(t-\tau)\left|\mathbf{h}^{k}(t) \mathbf{w}_{k}(t-\tau)\right|^{2}}{\sigma^{2}+\sum_{i \in \mathcal{K}, i \neq k} p_{i}(t-\tau)\left|\mathbf{h}^{k}(t) \mathbf{w}_{i}(t-\tau)\right|^{2}}
$$

The achievable rate of user $k$ at time $t$ is

$$
\mathrm{R}_{k}(t)=\log _{2}\left(1+\gamma_{k}(t)\right), \quad \forall k \in \mathcal{K}
$$


The system sum rate at time $t, R(t)$, is then obtained as

$$
R(t)=\sum_{k \in \mathcal{K}} \log _{2}\left(1+\gamma_{k}(t)\right)
$$

We formulate the sum rate maximization problem at time $t$ as follows:

$$
\begin{gathered}
\max _{\left\{\mathbf{w}_{k}\right\}} \sum_{k=1}^{K} \log _{2}\left(1+\gamma_{k}\left(\mathbf{w}_{k}\right)\right) \\
\text { s.t } \max _{j} \sum_{k=1}^{K}\left[\mathbf{w}_{k} p_{k} \mathbf{w}_{k}^{H}\right]_{j, j} \leq P \\
\gamma_{k}\left(\mathbf{w}_{k}\right) \geq \gamma_{0} \forall k
\end{gathered}
$$

where $P=\frac{P_{t}}{N_{t}}$ is the maximum transmit power of each RAU, $P_{t}$ is the total transmit power in the cell and $\gamma_{0}$ is target SINR of user $k$.

Assigning all RAUs to all the users may increase infeasibility due to increment of channel matrix dimension. This gives us a motivation of antenna selection. The problem (9) is difficult to solve directly due to i) non-convex cost function and constraint and ii) the computational complexity of designing a large precoding matrix. The cost function and constraint can be relaxed to be convex. However, the result may not reduce the computational complexity of optimization. Therefore, suboptimization needs to be established. One possible approach is to decompose it into multiple problems by antenna selection and user clustering to reduce the computational complexity.

To reduce the impact of residual interference on the system performance, the users are divided into groups based on its speed. Each group contains a set of users which has similar speeds. In the DAS, the user experiences different channels from RAUs due to different path loss. This motivates us to consider antenna selection among RAUs and select users by user clustering, which will reduce the system computational complexity.

\section{Cooperative Cluster Formation}

The cooperative cluster $c$ is formed to serve the set of users $\mathcal{C}_{c}$ while considering the non-negligible interference. The cooperation cluster formation consists of antenna selection and user clustering algorithms. The cluster formation is done after the channel estimation at the uplink transmit slot. The antenna selection and user clustering will be dynamic and will modify to adapt changes of the CSI.

\section{A. Antenna Selection}

Antenna selection is done at the CU based on the estimated channel. At the uplink transmit slot, the CU estimates channel for all users from all RAUs. At each stage, the $\mathrm{CU}$ assigns the RAU $j$ to the user $k$ based on the strongest channel gain of the estimated channel, given by

$$
j=\underset{j \in \mathcal{N}, k \in \mathcal{K}}{\arg \max }\left|h_{k, j}(t-\tau)\right|^{2}
$$

then the user $k$ and the RAU $j$ will be removed from allocation procedure. This procedure is repeated until all users are assigned to the RAUs.

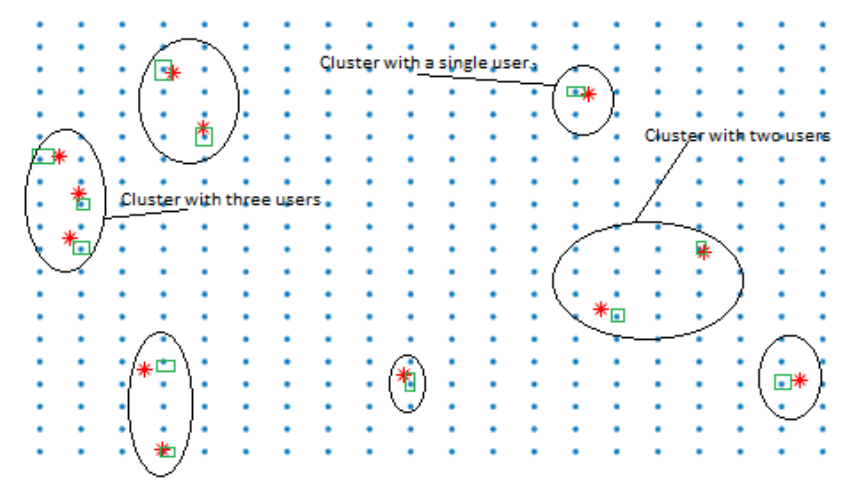

Fig. 3. Illustration of cooperative clustering for 12 users and 400 RAUs where clustering threshold is $20 \mathrm{~dB}$.

\section{B. User Clustering}

Each user has own mobility speeds. Based on user speed $(v)$, the users are classified into groups as follows:

$$
\begin{array}{lr}
0 \leq \text { speed } \leq v_{1} & \text { Low mobility } \\
v_{1}<\text { speed } \leq v_{2} & \text { Medium mobility } \\
v_{2}<\text { speed } \leq v_{3} & \text { High mobility }
\end{array}
$$

After the antenna selection, each group selects the first pair of user $k$ and its associated RAU which has the highest channel gain. The next user $i$ is chosen from the remaining unselected pairs and compute the SINR. The SINR is calculated under the assumption of maximum power transmission and maximum ratio combining [11] and given as:

$\gamma_{k, i}=\min \left\{\frac{\sum_{j \in \mathcal{N}_{k}}\left|h_{k, j}\right|^{2} P}{\sigma^{2}+\sum_{j^{\prime} \in \mathcal{N}_{i}}\left|h_{k j^{\prime}}\right|^{2} P}, \frac{\sum_{j^{\prime} \in \mathcal{N}_{i}}\left|h_{i j^{\prime}}\right|^{2} P}{\sigma^{2}+\sum_{j \in \mathcal{N}_{k}}\left|h_{i j}\right|^{2} P}\right\}$

where $\left|\mathcal{N}_{k}\right|$ is a set of RAUs to serve the user $k$.

If the minimum SINR between the selected user and the chosen user $\left(\gamma_{k, i}\right)$ is less or equal to $\gamma_{c}$, i.e., $\gamma_{k, i} \leq \gamma_{c}$, then the chosen user is added to the cluster ,i.e., $\mathcal{C}_{c} \cup i$. This process is repeated until all the pairs of user-RAU are assigned to the cluster.

The performance of the system is severely degraded due to uncoordinated inter-group-inter-cluster interference. If the SINR of two interfering neighbouring clusters are smaller than the cluster SINR threshold, then the neighbouring clusters are merged and form a cooperative cluster, as shown as fig 3 .

\section{Cluster based sub-problem formation}

Once we complete the user clustering, the inter cluster interferences are suppressed, i.e., $\gamma_{k, i} \leq \gamma_{c}$. The SINR (6) of $k$-th user of the $c$-th cluster at time $t$ is rewritten as

$$
\gamma_{k, c}(t)=\frac{p(t-\tau)\left|\mathbf{h}_{c, c}^{k}(t) \mathbf{w}_{k, c}(t-\tau)\right|^{2}}{\sigma^{2}+\sum_{i \neq k} p(t-\tau)\left|\mathbf{h}_{c, c}^{k}(t) \mathbf{w}_{i, c}(t-\tau)\right|^{2}+\mathrm{I}_{\text {inter }}}
$$

where $\mathrm{I}_{\text {inter }}=\sum_{j \in C, j \neq c}\left|\mathbf{h}_{c, j}^{k}(t) \mathbf{W}_{j}(t-\tau) \sqrt{\mathbf{P}_{j}(t-\tau)}\right|^{2}$, $\mathbf{h}_{c, c}^{k} \in \mathbb{C}^{1 \times\left|\mathcal{N}_{c}\right|}$ is a channel vector from $\mathrm{RAU}_{c}$ to the $k$-th user of the $c$-th cluster, $\mathbf{w}_{k, c} \in \mathbb{C}^{\left|\mathcal{N}_{c}\right| \times\left|\mathcal{C}_{c}\right|}$ denotes the precoding vector for the $c$-th cluster, $\mathbf{P}_{j} \in \mathbb{C}^{\left|\mathcal{C}_{j}\right| \times\left|\mathcal{C}_{j}\right|}$ is a diagonal matrix of power normalization factor of each RAU. We assumed same 
power normalization factor $p$ for every RAU lies in the same cluster.

Hence, the RAU selection and user clustering gives subproblem formulation that maximizes the sum rate at time $t$ and gives as:

$$
\begin{aligned}
& \max _{\left\{\mathbf{w}_{k, c}\right\}} \sum_{k \in \mathcal{C}_{c}} \log _{2}\left(1+\gamma_{k, c}\left(\mathbf{w}_{k, c}\right)\right) \\
\text { s.t } & \max _{j} \sum_{k \in \mathcal{C}_{c}}\left[\mathbf{w}_{k, c} p_{k} \mathbf{w}_{k, c}^{H}\right]_{j, j} \leq P \\
& \gamma_{k}\left(\mathbf{w}_{k, c}\right) \geq \gamma_{0} \quad \forall k
\end{aligned}
$$

The number of users within a cluster depends on the cluster SINR threshold. If the cluster SINR threshold is small, then the system forms single-user cluster with high probability. In the single-user cluster, a pair of user and RAU is selected which has the highest channel gain. If the cluster SINR threshold is high, then the system formed one cluster with high probability. In the one cluster, all users are jointly served using MU-MIMO precoding.

\section{Feedback Interval Allocation}

The aim of feedback time slot allocation for the cluster to minimize the channel mismatch error. The feedback model and the frame structure of the TDD system as shown in fig. 1 . The channel matrix remains same during a time slot (with length $T_{c}$ ). The time frame (with length $T$ ) between two consequent channel updates is called feedback interval (with length $T_{f}$ ). For simplicity, we assume there are $\mathrm{N}$ (an integer) time slots in a time frame, i.e., $T=N T_{c}$ and first time slot is always use for uplink pilot.

When the mobility speed increases or the transmission delay increases, the system performance severely degrade due to channel mismatch error. This motivates us to calculate the feedback interval of the mobility user based on the autocorrelation of the channel. The autocorrelation, $R(\tau)$, of the channel is equal to the zeroth order Bessel function of the first kind, $J_{0}($.$) , as,$

$$
R(\tau)=J_{0}\left(2 \pi f_{d} \tau\right)
$$

where $f_{d}$ is the maximum Doppler shift and $\tau$ is a delay, i.e., $\tau=n T_{c}, n=\{1, \cdots, \cdots, N\}$. As mentioned above, a long feedback interval limits the system performance. In order to overcome the problem, we introduce a minimum autocorrelation coefficient $\rho_{o}$. Therefore, when $R(\tau)<\rho_{o}$, $n T_{c}$ becomes next consequent uplink time slot. The feedback interval $\left(T_{f}\right)$ of the user becomes,

$$
T_{f}=(n-1) T_{c}, \text { where } n=\{1, \cdots, \cdots, N\}
$$

Let $N_{u}$ and $N_{d}$ be number of uplink/feedback time slots and number of downlink time slots respectively, which are given as:

$$
N_{u}=\left\lceil\frac{N T_{c}}{T_{f}}\right\rceil \text { and } N_{d}=N-N_{u}
$$

Let $T_{u}$ be uplink/feedback time slot of time frame $T$ and given as:

$$
T_{u}=1+\left\lfloor(m-1) \frac{N}{N_{u}}\right\rfloor, \text { where } m=\left\{1, \cdots, \cdots, N_{u}\right\}
$$

In the cooperative clustering, each cluster may have user with different range of mobility. Therefore, the feedback time slot of the cooperative cluster is allocated based on the fastest mobility user, to minimize the mismatch error of the high mobility user.

\section{Simulation Results}

We consider a TDD downlink MU-DAS system. We assume that the users have different mobility and its speed range is 0 to $15 \mathrm{~m} / \mathrm{s}$. We assume that the MSI is perfectly known at the CU. The period of the TDD radio frame is assumed as $T=10$ ms, where the radio frame is divided into $N=100$ time slots or sub-frames. In the antenna selection, the number of RAUs assigns to user is one. Table I summarizes the MU-DAS system parameters for performance evaluation.

TABLE I

Simulation PARAMETERS

\begin{tabular}{|l|l|}
\hline Parameter Settings & Value \\
\hline Cell Model & square grid1 km² \\
Carrier Frequency & $2 \mathrm{GHz}$ \\
Number of RAUs & 400 \\
Intra RAU distance & $50 \mathrm{~m}$ \\
Number of users & from 2 to 4 \\
Users distribution & Uniform \\
Min dist. between RAU and user & from $10 \mathrm{~m}$ \\
Path loss exponent & 3 \\
Number of scatterers & 64 \\
Radio frame duration & $10 \mathrm{~ms}$ \\
Time slot duration & $0.1 \mathrm{~ms}$ \\
Total transmit power $\left(P_{t}\right)$ & $46 \mathrm{dBm}$ \\
Noise power & $-104 \mathrm{dBm}$ \\
User speed range & $0 \mathrm{~m} / \mathrm{s} \mathrm{to} 20 \mathrm{~m} / \mathrm{s}$ \\
Cluster SINR threshold & $20 \mathrm{~dB}$ \\
User target SINR & $50 \mathrm{~dB}$ \\
Minimum autocorrelation & 0.8 \\
\hline
\end{tabular}

Assuming the minimum autocorrelation coefficient $\rho_{o}=$ 0.8 , the number feedback time slots $N_{u}$ and the number of downlink time slots $N_{d}$ is calculated based on (17). Fig.4 illustrates the feedback interval of different mobility users as a function of time delay. The $R(\tau)$ of user with speed below $2 \mathrm{~m} / \mathrm{s}$ has always been higher than $\rho_{o}$. When users speed increases or transmission delay increases, the $R(\tau)$ becomes less than $\rho_{o}$. So, the next time slot becomes uplink time slot. Therefore, the $R(\tau)$ of user with speed $4 \mathrm{~m} / \mathrm{s}$ becomes high when transmission delay is $5.1 \mathrm{~ms}$, i.e., $T_{f}=5.0 \mathrm{~ms}, n=50$. Table II summarizes the uplink/feedback and downlink parameters for performance evaluation.

TABLE II

FEEDBACK PARAMETERS

\begin{tabular}{|l|l|l|l|}
\hline Speed $(\mathrm{m} / \mathrm{s})$ & $N_{u}$ & $N_{d}$ & $T_{u}$ \\
\hline $0-2$ & 1 & 99 & $1^{s t}$ \\
$2-4$ & 2 & 98 & $1^{s t}, 51^{s t}$ \\
$4-6$ & 3 & 97 & $1^{s t}, 35^{t h}, 69^{t h}$ \\
$6-9$ & 4 & 96 & $1^{s t}, 26^{t h}, 51^{s t}, 76^{t h}$ \\
$9-11$ & 5 & 95 & $1^{s t}, 21^{s t}, 41^{s t}, 61^{s t}, 81^{s t}$ \\
$11-13$ & 6 & 94 & $1^{s t}, 18^{t h}, 35^{t h}, 52^{t h}, 69^{t h}, 86^{t h}$ \\
$13-15$ & 7 & 93 & $1^{s t}, 16^{t h}, 31^{s t}, 46^{t h}, 76^{t h}, 91^{s t}$ \\
\hline
\end{tabular}




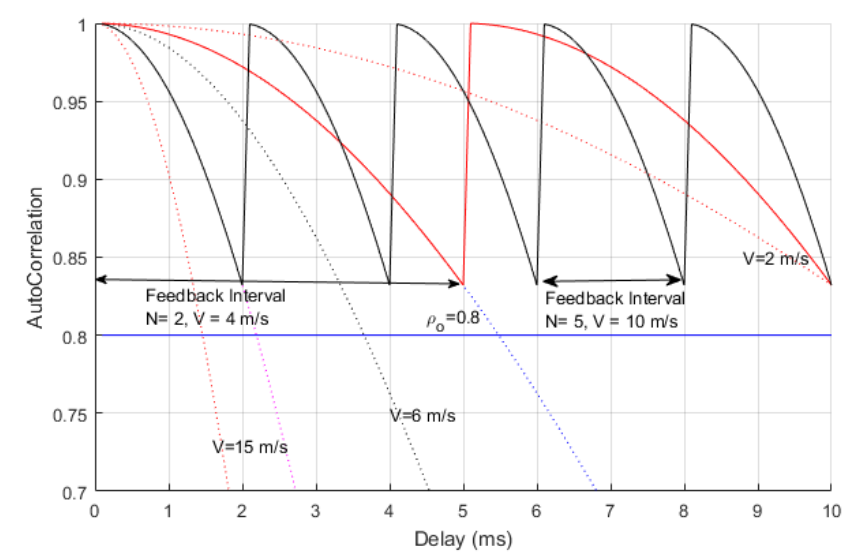

Fig. 4. Illustration of feedback interval of different mobility users, where $\rho_{o}=0.8$.

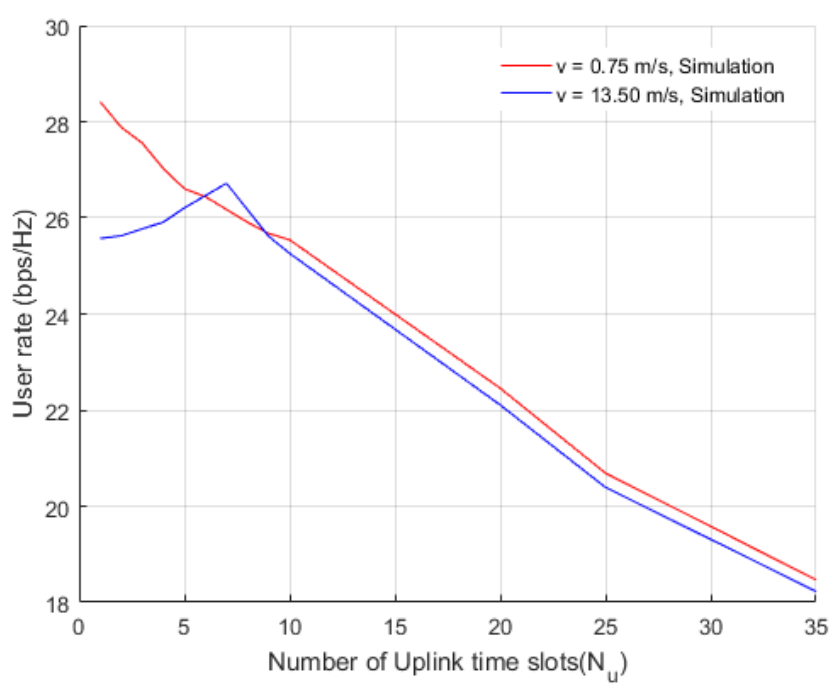

Fig. 5. Illustration of Average user rate of different mobility users over number of uplink time slots, $N_{u}$, where $\gamma_{c}=-60 d B, \rho_{o}=0.8, K=2$ and $N_{t}=400$.

In Fig. 5 illustrates the average user rate of single user clustering as a function of the number of uplink time slots $N_{u}$ with fixed user speed. When $v=0.75 \mathrm{~m} / \mathrm{s}$, the autocorrelation coefficient of user is always higher than threshold value because the channel mismatch error is small within the feedback interval. Therefore, the rate is highest at the least number of uplink time slots, i.e., $N_{u}=1$. Howevere, the rate starts to decrease when the number of uplink time slots increases due to reduction of the downlink time slots in the overall time slots. When $v=13.50 \mathrm{~m} / \mathrm{s}$, the channel mismatch becomes large within feedback interval and the autocorrelation becomes less than threshold value. Therefore, up to $N_{u}=7$, the user rate starts to increase due to frequent CSI update. However, when $N_{u}>7$, the CSI is more frequently updated at the cost of the downlink time slots in overall time slots. Therefore, the user rate starts to decrease when the number of uplink time slots is more than seven, i.e., $N_{u}>7$.

Fig. 6 illustrates the average sum rate as a function of speed. At low clustering threshold, all the clusters become the single user cluster with high probability. The single user cluster consists of one pair of the user and the RAU. The

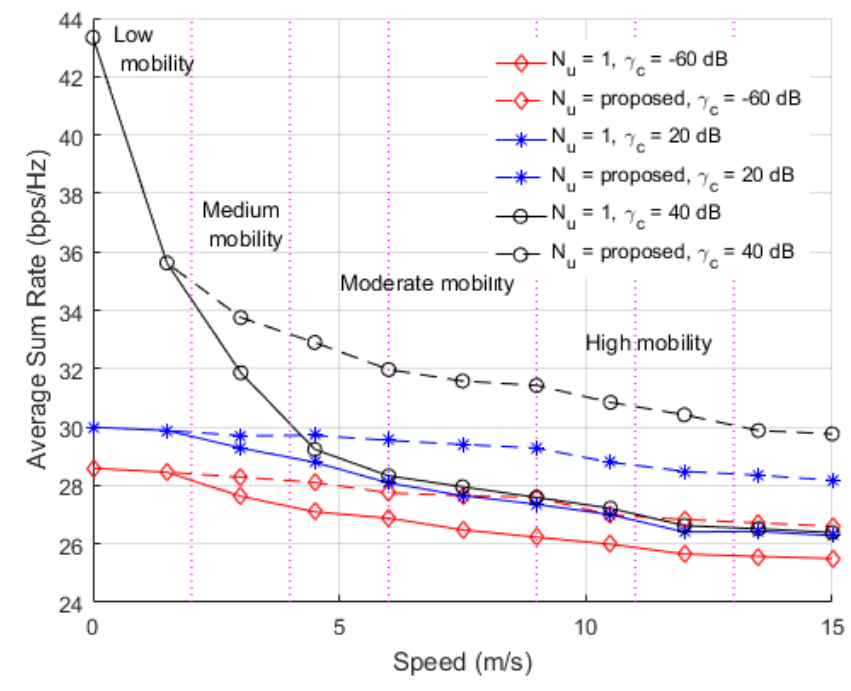

Fig. 6. Illustration of Average sum rate over changing mobility of the users, where $\rho_{o}=0.8, K=2$ and $N_{t}=400$.

inter cluster interference becomes the main limiting factor to limit the system sum rate. As the clustering threshold increases, the multiple cooperative clustering is formed, where multiple users are jointly processed to cancel inter group-inter cluster interference. At the high clustering threshold regime, the system forms one cooperative cluster with high probability including all users. The feedback time slot of the cluster is allocated based on the fastest mobility user, to minimize the mismatch error. Thus, the noise is only limiting factor and improved the average sum rate. In low mobility group, the users are in walking speed or at stationary. The residual interference remains small even though the transmission delay increases. Therefore, the performance of proposed technique and least number of uplink time slot remain same. When user mobility increases, the residual interference increases as the transmission delay increases. The average sum rate becomes less than the average sum rate of the low mobility group due to channel mismatch error. By reducing the feedback interval, the CSI is updated regularly and reduces the residual interference. Therefore, the average sum rate of proposed technique outperforms a system with least number of feedback interval, i.e., $N_{u}=1$ at any clustering threshold regime.

Fig. 7 illustrates the average user rate as a function of autocorrelation threshold. The autocorrelation of user speed up to $2 \mathrm{~m} / \mathrm{s}$ is always high due to slow channel variation. When user speed increases, the proposed scheme is used to reduce the channel mismatch error. When $v=15 \mathrm{~m} / \mathrm{s}$, the user rate increases up to $R(\tau)=0.6$ due to higher correlation value or less channel mismatch error. After $R(\tau)<0.6$, the channel mismatch error is reduced due to decrement of feedback interval or increment of the uplink time slot. However, the rate starts to decrease due to reduction of the number of downlink time slots in the overall time slots. Thus, fig. 7 shows the optimal value of autocorrelation threshold at single user clustering.

\section{CONCLUSION}

This paper has studied the sum rate of TDD downlink MUDAS with the consideration of different user speed. A feedback 


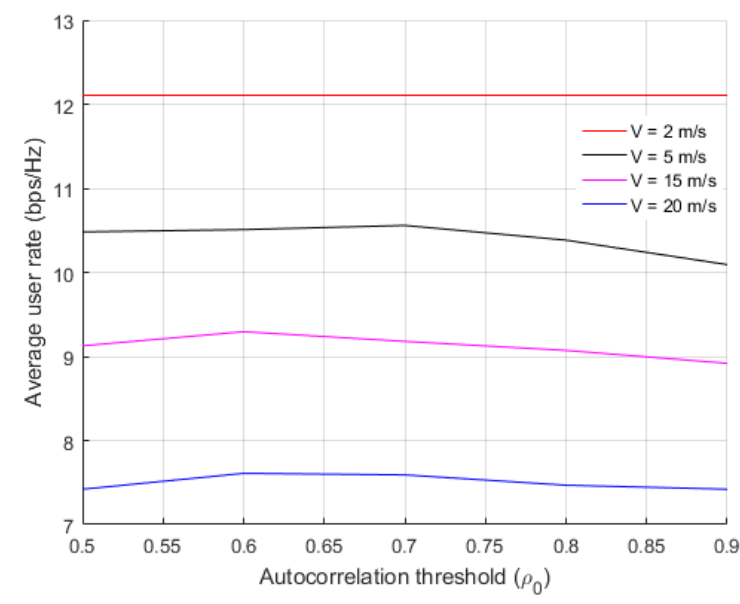

Fig. 7. Illustration of Average user rate over autocorrelation threshold, where $\gamma_{c}=-60 d B, K=4$ and $N_{t}=400$.

interval reduction technique based on an autocorrelation of the channel is proposed to minimize the channel mismatch error. The channel gain based antenna selection and SINR threshold based user clustering is proposed to reduce the system computational complexity. To maximize the sum rate, the cooperative clustering is proposed where the feedback interval technique is implemented based on fastest mobility user. The numerical results have shown that the proposed technique can maximize the system sum rate in user movement environment. The numerical result has also shown that individual autocorrelation threshold value can be allocated to each cluster, to maximize the system sum rate. The proposed technique has good performance for wide range of speed and suitable for future wireless communication systems.

\section{ACKNOWLEDGMENT}

This work has received funding from the European Union's Horizon 2020 Research and Innovation programme under grant agreement no. 643297 (RAPID) and Institute for Infocomm Research, A`STAR, Singapore.

\section{REFERENCES}

[1] A. Khadka, K. Adachi, S. Sun, H. Zhu and J. Wang, "Cooperative Transmission Strategy for Downlink Distributed Antenna Systems Over Time-Varying Channel", in Proc. IEEE Globecom, Dec., 2015.

[2] H. Zhu, "Radio resource allocation for OFDMA systems in high speed environments," IEEE J. Sel. Areas Commun., vol. 30, pp. 748-759, May 2012.

[3] H. Zhu and J. Wang, "Chunk-based resource allocation in OFDMA systems - Part II: joint chunk, power and bit allocation," IEEE Trans. Commun., vol. 60, no. 2, pp. 499-509, Feb. 2012.

[4] H. Zhu, "On frequency reuse in cooperative distributed antenna systems", IEEE Commun. Magazine, vol. 50, no. 4, pp.85-89, Apr. 2012.

[5] H. Zhu, S. Karachontzitis and D. Toumpakaris, "Low-complexity resource allocation and its application to distributed antenna systems," IEEE Wireless Commun. Magazine, vol. 17, no. 3, pp. 44-50, Jun. 2010.

[6] X. You, et al, "Cell edge performance of cellular mobile systems," IEEE J. Sel. Areas Commun., vol. 29, no. 6, pp. 1139-1150, Jun. 2011.

[7] H. Zhu, "Performance comparison between distributed antenna and microcellular systems," IEEE J. Sel. Areas in Commun., vol. 29, no. 6, pp. 1151-1163, Jun. 2011.

[8] L. Dai, "A comparative study on uplink sum capacity with co-located and distributed antennas," IEEE J. Sel. Areas Commun., vol. 29, no. 6, pp. 1200-1213, Jun. 2011.
[9] J. Wang, H. Zhu and N. J. Gomes, "Distributed antenna aystems for mobile communications in high speed trains," IEEE J. Sel. Areas in Commun., vol. 30, no. 4, pp. 675-683, Apr. 2012.

[10] H. Zhu and J. Wang, "Radio resource allocation in multiuser distributed antenna systems," IEEE J. Sel. Areas in Commun., vol. 31, no. 10, pp. 2058-2066, Oct. 2013.

[11] J. Joung, Y. K. Chia and S. Sun, "Energy-efficient, large-scale distributed-antenna system (L-DAS) for multiple users," IEEE J. Sel. Top. Sign. Proces., vol. 8, no. 5, pp. 954-965, Oct. 2014.

[12] A. Wiesel, Y. C. Eldar and S. Shami, "Zero-Forcing precoding and generalized inverses," IEEE Trans. Signal Processing, vol. 56, no. 9, pp. 4409-4418, Sept. 2008.

[13] B, Bandemer, M, Haardt and S, Visuri, "Linear MMSE Multi-User MIMO Downlink Precoding for Users with Multiple Antennas," Personal, Indoor and Mobile Radio Communications, pp. 1-5, Sept. 2006.

[14] I. Sohn, K. B. Lee and Y. S. Choi, "Comparison of decentralized time slot allocation strategies for asymmetric traffic in TDD systems," IEEE Trans. Wireless Commun., vol. 8, no. 6, pp. 2990-3003, Jun. 2009.

[15] P. Komulainen, A. Tolli and M. Juntti, "Effective CSI signaling and decentralized beam coordination in TDD multi-cell MIMO systems," IEEE Trans. Sign. Proces., vol. 61, no. 9, pp. 2204-2218, May 2013.

[16] T. L. Marzetta and B. M. Hochwald, "Fast transfer of channel state information in wireless systems," IEEE Trans. Sig. Proces., vol. 54, no. 4, pp. 1268-1278, 2006.

[17] S. Pandula and B-P. Paris, "Performance analysis of MIMO receivers under imperfect CSIT," IEEE Inform. Sciences and Systems, pp. 107-112, Mar. 2007.

[18] G. Primolevo, O. Simeone and U. Spagnolini, "Effects of imperfect channel state information on the capacity of broadcast OSDMA-MIMO systems," IEEE Workshop on Sig. Proc. Advances in Wireless Commun., pp. 546-550, Jul. 2004.

[19] H. Shirani-Mehr, G. Caire and M. J. Neely, "MIMO downlink scheduling with non-perfect channel state knowledge," IEEE Trans. Commun., vol. 58, no. 7, pp. 2055-2066, Jul. 2010.

[20] J. So and J.M. Cioffi, "Feedback reduction scheme for downlink multiuser diversity," IEEE Tarns. Wireless Commun., vol. 8, no. 2, pp. 668672, Feb. 2009.

[21] M. Bashar, M. Eslami and M. J. Dehghani, "Threshold-based CSI feedback reduction for time-varying multiple-input multiple-output broadcast channels," IET Commun., vol. 8, no. 9, pp. 1616-1625, Feb. 2014.

[22] P. Dent, G. E. Bottomley and T. Croft, "Jakes fading model revisited," IEEE Electron. lett., vol.29, no.13, pp. 1162-1163, Jun. 1993.

[23] C. Wang and R. D. Murch,"Adaptive downlink multi-user MIMO wireless systems for correlated channels with imperfect CSI," IEEE Trans. Wireless Commun., vol. 5, no. 9, pp. 2435-2446, Sept. 2006.

[24] R.H. Clarke, "A statistical theory of mobile radio reception," Bell Syst. Tech. J., pp. 957-1000, Jul. 1968. 